\title{
Breakfast Omission Reduces Subsequent Resistance Exercise Performance
}

\author{
Mohamed Nashrudin Bin Naharudin, ${ }^{1,2}$ Ashril Yusof,${ }^{2}$ Harky Shaw, ${ }^{1}$ Matthew Stockton, ${ }^{1}$ \\ David J. Clayton, ${ }^{3}$ and Lewis J. James ${ }^{1}$ \\ ${ }^{1}$ National Center of Sport and Exercise Medicine, School of Sport Exercise and Health Science, Loughborough University, \\ Leicester, United Kingdom; ${ }^{2}$ Sport Center, University Malaya, Kuala Lumpur, Malaysia; and ${ }^{3}$ School of Science and \\ Technology, Nottingham Trent University, Nottingham, United Kingdom
}

\begin{abstract}
Bin Naharudin, MN, Yusof, A, Shaw, H, Stockton, M, Clayton, DJ, and James, $\amalg$. Breakfast omission reduces subsequent resistance exercise performance. J Strength Cond Res XX(X): 000-000, 2019 -Although much research has examined the influence of morning carbohydrate intake (i.e., breakfast) on endurance performance, little is known about its effects on performance in resistance-type exercise. Sixteen resistance-trained men (age $23 \pm 4$ years, body mass $77.56 \pm 7.13 \mathrm{~kg}$, and height $1.75 \pm 0.04 \mathrm{~m})$ who regularly $(\geq 3$ day/wk ${ }^{-1}$ ) consumed breakfast completed this study. After assessment of 10 repetition maximum (10RM) and familiarization process, subjects completed 2 randomized trials. After an overnight fast, subjects consumed either a typical breakfast meal (containing 1.5 $\mathrm{g}$ of carbohydrate/kg; breakfast consumption [BC]) or a water-only breakfast (breakfast omission [BO]). Two hours later, subjects performed 4 sets to failure of back squat and bench press at $90 \%$ of their 10RM. Sensations of hunger, fullness, desire to eat, and prospective food consumption were collected before, as well as immediately, 1 hour and 2 hours after BC/BO using 100-mm visual analogue scales. Total repetitions completed were lower during BO for both back squat (BO: $58 \pm 11$ repetitions; $B C: 68 \pm 14$ repetitions; effect size $[\mathrm{ES}]=0.98 ; p<0.001$ ) and bench press (BO: $38 \pm 5$ repetitions; $\mathrm{BC}: 40 \pm 5$ repetitions; $\mathrm{ES}=1.06 ; p<$ $0.001)$. Fullness was greater, whereas hunger, desire to eat, and prospective food consumption were lower after a meal for BC compared with BO $(p<0.001)$. The results of this study demonstrate that omission of a pre-exercise breakfast might impair resistance exercise performance in habitual breakfast consumers. Therefore, consumption of a high-carbohydrate meal before resistance exercise might be a prudent strategy to help maximize performance.
\end{abstract}

KEY WoRDs fasting, resistance training, strength training, hypertrophy

Address correspondence to Lewis J. James, L.James@lboro.ac.uk.

$00(00) / 1-7$

Journal of Strength and Conditioning Research

(c) 2019 National Strength and Conditioning Association

\section{INTRODUCTION}

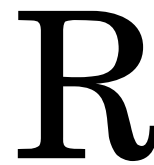

esistance exercise is performed by many athletes, often as part of a wider training program, with performance in such sessions having potential implications for adaptation to the resistance exercise itself and possibly for other aspects of the athlete's performance (e.g., sport-specific strength performance) or health (e.g. injury prevention/recovery). High-intensity exercise largely relies on the utilization of endogenous carbohydrate (glycogen) stores to supply energy for muscular contraction, and as such, current recommendations suggest consuming $1-4 \mathrm{~g}$ of carbohydrate $/ \mathrm{kg}$ body mass in the $1-4$ hours before exercise (2). Although much research has examined the influence of pre-exercise carbohydrate intake (29) and breakfast (6) on endurance exercise performance, little is known about how pre-exercise carbohydrate intake affects performance in resistance-type exercise.

Glycogen depletion of up to $40 \%$ has been reported after a single bout of resistance exercise $(12,13,30,32)$. The degree of glycogen depletion is likely dependent on the type, intensity, and duration of the exercise session, with hypertrophytype resistance exercise (i.e., higher repetition and moderate load exercise) likely to produce larger reductions in muscle glycogen (31). Previous studies have demonstrated that commencing resistance-type exercise with suboptimal muscle glycogen levels can impair performance capabilities $(17,23)$. Although this is not a universal finding (25), muscle glycogen seems to be an important fuel source for resistance exercise performance, and pre-exercise glycogen stores might therefore affect performance and possible training quality/ adaptation.

Morning training sessions are common place for both athletes and recreational exercisers, and many athletes report skipping breakfast in the morning $(33,35)$. Therefore, it is likely that at least some training will take place in a fasted state, where glycogen levels (at least liver glycogen) will be suboptimal. Consumption of a high-carbohydrate meal (i.e., breakfast) in the morning after an overnight fast increases liver (1) and, to a less extent, muscle $(3,7)$ glycogen levels and has been shown to enhance endurance performance $(26,29)$. By contrast, the effect of morning 
TABLE 1. Macronutrient, energy, and water intake during each trial. ${ }^{*} \dagger$

\begin{tabular}{|c|c|c|}
\hline & \multicolumn{2}{|c|}{ Breakfast meal } \\
\hline & $\mathrm{BO}$ & $\mathrm{BC}$ \\
\hline Protein (g) & $0 \pm 0$ & $14.7 \pm 1.4$ \\
\hline Carbohydrate (g) & $0 \pm 0$ & $116.3 \pm 10.7$ \\
\hline Fat $(\mathrm{g})$ & $0 \pm 0$ & $8.4 \pm 0.8$ \\
\hline Fiber (g) & $0 \pm 0$ & $3.8 \pm 0.4$ \\
\hline Energy (kcal) & $0 \pm 0$ & $600 \pm 55$ \\
\hline Water (ml) & $514 \pm 72$ & $514 \pm 72$ \\
\hline \multicolumn{3}{|c|}{$\begin{array}{l}{ }^{*} \mathrm{BO}=\text { breakfast omission; } \mathrm{BC}=\text { breakfast consump- } \\
\text { tion. } \\
+ \text { Values are mean } \pm S D, n=16 .\end{array}$} \\
\hline
\end{tabular}

carbohydrate intake before resistance exercise has not been well studied.

Carbohydrate intake before and during a bout of resistance exercise increases $(14,15)$ or tends to increase (22) performance when many sets of $\sim 10$ repetitions are performed to exhaustion. By contrast, carbohydrate intake before or during more intense but lower volume (i.e., sets of 5 repetitions) resistance exercise (20) or fixed repetition (3 sets of 10 repetitions) isokinetic dynamometer exercise (12) does not seem to influence performance. These studies suggest that carbohydrate ingestion around resistance exercise is more likely to augment performance when the volume of the training session is greater.

It is not possible to isolate the specific effects of preexercise carbohydrate feeding in these experiments because carbohydrate (and placebo) drinks were provided both before and during exercise. The only study, to the best of our knowledge, to isolate the effect of pre-exercise carbohydrate intake on resistance exercise performance reported no benefit of carbohydrate intake on performance of 3 maximal efforts on an isokinetic dynamometer, for up to 90 minutes after ingestion (8). However, given the findings of previous studies, the low-volume nature of the exercise performed in this study might not be expected to be influenced by carbohydrate intake or manipulation of pre-exercise glycogen stores. In addition, although the provision of carbohydrate in drink form might allow the impact of nutrient ingestion to be elucidated, it might not represent a typical breakfast consumed before exercise. Therefore, the purpose of this study was to examine the effect of a typical high-carbohydrate breakfast meal on subsequent performance of 4 sets of resistance exercise at 90\% 10 repetition maximum $(10 \mathrm{RM})$ to failure (squat and bench press exercise). It was hypothesized that breakfast omission (BO) would decrease the performance.

\section{Methods}

\section{Experimental Approach to the Problem}

To investigate the problem, subjects, in a cross-over fashion, consumed a typical breakfast meal or a water-only breakfast 2 hours before completing 4 sets of back squat and bench press, with each set performed to failure. The number of repetitions performed in each set was recorded during both trials to quantify performance.

\section{Subjects}

Sixteen resistance-trained men (mean $\pm S D$ : age $23 \pm 4$ years, body mass $77.56 \pm 7.13 \mathrm{~kg}$, height $1.75 \pm 0.04 \mathrm{~m}$, and body mass index $25.3 \pm 2.3 \mathrm{~kg} / \mathrm{m}^{2}$ ) completed this study. The study received ethical approval from the Loughborough University Ethical Approvals (Human Participants) Sub-Committee. Subjects completed a medical screening questionnaire before any data collection and provided written and verbal consent before commencing the study. To participate in the study, subjects were required to be nonsmokers, habitually consuming breakfast $\geq 3$ times a week, and habitually performing resistance exercise $\geq 2$ times a week for at least 2 years, and required to include back squat and bench press in their training routine. On average, subjects consumed breakfast $5 \pm 2$ times per week and performed resistance exercise $4 \pm 2$ times per week, with back squat and bench press performed $2 \pm 1$ times per week and $2 \pm 1$ times per week, respectively. The sample size for this study was estimated from $G^{*}$ Power 3.0.10 software using an $\alpha$ of 0.05 , statistical power of 0.95 , and estimated correlation between-group of 0.5 . It was estimated that 16 subjects would be sufficient to detect a $10 \%$ difference in performance between trials.

\section{Procedures}

Pre-trial Standardization. In the 24 hours before the first experimental trial, subjects weighed and recorded all dietary intakes. Subjects also recorded all low-intensity habitual activity for the 48 hours before the first trial and any physical activity undertaken between 48 and 72 hours before the first trial. These diet and activity patterns were replicated in the 72 hours before the second experimental trial. Subjects refrained from any strenuous exercise or alcohol intake in the 48 hours before trials.

Preliminary/Familiarization Trial. At the preliminary trial, subjects were briefed on the study, before completing consent and screening forms. Subjects' physical characteristics (body mass and height) were then measured before their 10RM was determined for 2 bilateral resistance exercises (back squat and bench press). Before exercise, subjects performed a 5-minute cycling warm-up at $1.5 \mathrm{~W} / \mathrm{kg}$ body mass (Monark 894E; Monark Exercise AB, Vansbro, Sweden). They then completed the 10RM testing in back squat, followed by bench press, each of which was preceded by a 5minute self-selected exercise-specific warm-up. Subjects completed 10 repetitions of the exercise, with the load 
continuing to increase until they were unable to complete 10 repetitions. The last completed set was termed the subjects' $10 \mathrm{RM}$ and was used to determine the workload for familiarization and experimental trials, nominally $90 \%$ of $10 \mathrm{RM}$. After the $10 \mathrm{RM}$ testing and 30 minutes of rest, subjects were familiarized with the exercise component of the experimental trials and the measurement of subjective appetite (both described in detail below).

Experimental Trials. For experimental trials, subjects arrived at the laboratory after an overnight fast $(\sim 10$ hours $)$ at a time typical for them to consume breakfast (i.e., $\sim 0800-0900$ ), and baseline measurements of body mass and subjective appetite were recorded.

During the breakfast consumption (BC) trial, subjects consumed a standardized breakfast meal consisting of rice cereal, milk, bread, butter, jam, and orange juice (Tesco, Welwyn Garden City, United Kingdom) providing 20\% of their estimated energy requirements and $1.5 \mathrm{~g}$ of carbohydrate $/ \mathrm{kg}$ body mass. During BO, no food was consumed, but subjects consumed water to match the total water content of the $\mathrm{BC}$ meal. Meals were consumed over 10 minutes and were followed by 2-hour seated rest in the laboratory. The energy and macronutrient content of the breakfasts is provided in Table 1 .

Resistance Exercise Performance. To assess resistance exercise performance, subjects performed 4 sets to failure of both back squat and bench press at $90 \%$ of each subject's 10RM. Subjects initially performed a 5-minute cycling warm-up, as previously described. For each exercise, subjects then performed 5 minutes of individually standardized self-selected stretching and exercise-specific warm-up, followed by 2 warm-up sets of 10 repetitions at 30 and $60 \%$ of $10 \mathrm{RM}$. All exercise sets were followed by 3 -minute rest. For each exercise, subjects performed a standardized lifting technique, with 2 spotters assisting them to reach the starting position for each set. For the squat, the bar was held across the back of subjects' shoulders, and they started with knees fully extended, lowering themselves until their thighs were parallel to the floor, before returning to the starting position. For bench press, subjects started with their elbows fully extended, and they lowered the bar until it lightly touched their chest, before returning to the starting position. Each correctly completed repetition was counted by an investigator, and failure was defined as when subjects were unable to complete a repetition.

Subjective Appetite Sensations. Subjects rated their sensations of hunger, fullness, desire to eat (DTE), and prospective food consumption (PFC) using a visual analogue scale before $\mathrm{BC} /$ BO $(\sim 08: 20)$, after $\mathrm{BC} / \mathrm{BO}(\sim 08: 30)$, as well as 1 hour $(\sim 09: 30)$ and 2 hours $(\sim 10: 30)$ after BC/BO. Written anchors of "not at all"/"none at all" and "extremely"/"a lot" were placed 0 and $100 \mathrm{~mm}$, respectively (9).

\section{Statistical Analyses}

Data are reported as mean and $S D(\mathrm{M} \pm S D)$ and were analyzed using SPSS software (Version 23.0; IBM, Corp., Armonk, NY). The Shapiro-Wilk test was initially used to determine the normality of data. Data containing 2 factors were analyzed using 2-way repeated-measures analysis of variance, with significant effects followed by the Holm-Bonferroniadjusted paired $t$-test or Holm-Bonferroni-adjusted Wilcoxon signed-rank test, as appropriate. Data containing one factor were analyzed using the paired $t$-test or Wilcoxon signed-rank test, as appropriate. Cohen's d effect size (ES) was calculated for single-factor comparisons, whereas $\eta_{\mathrm{p}}^{2}$ was determined for comparisons containing 2 factors. In addition, confidence intervals (CIs) were calculated, as appropriate. Statistical significance was set at $p \leq 0.05$.

\section{Results}

\section{Pre-trial Measures and Breakfast Perceptions}

Pre-trial body mass was similar between trials (BO: $77.78 \pm$ $7.33 \mathrm{~kg}$; BC: $77.58 \pm 7.21 \mathrm{~kg}$; ES $=0.30$; $p=0.244)$. Subjective feelings of hunger $(\mathrm{ES}=0.47 ; p=0.083$ ), fullness (ES $=0.50 ; p=0.245)$, DTE $(\mathrm{ES}=0.06 ; p=0.088)$, and PFC (ES $=0.05 ; p=0.775$ ) were also similar between trials (Table 2).

TABLE 2. Subjects' appetite sensations of hunger, fullness, desire to eat (DTE), and prospective food consumption (PFC). ${ }^{*} \dagger$

\begin{tabular}{lll}
\hline Appetite sensation & \multicolumn{1}{c}{ BO } & BC \\
\hline Hunger (mm) & & \\
$\quad$ Pre-meal & $62 \pm 17$ & $51 \pm 20$ \\
Post-meal & $63 \pm 20 \ddagger$ & $12 \pm 9 \S$ \\
1 hour after meal & $72 \pm 17 \ddagger$ & $31 \pm 19 \S$ \\
2 hours after meal & $78 \pm 15 \ddagger \S$ & $45 \pm 27$ \\
Fullness (mm) & & \\
$\quad$ Pre-meal & $25 \pm 21$ & $30 \pm 18$ \\
Post-meal & $44 \pm 23 \ddagger \S$ & $81 \pm 10 \S$ \\
1 hour after meal & $24 \pm 15 \ddagger$ & $62 \pm 17 \S$ \\
2 hours after meal & $19 \pm 14 \ddagger$ & $45 \pm 22 \S$ \\
DTE (mm) & & \\
Pre-meal & $67 \pm 11$ & $61 \pm 19$ \\
Post-meal & $65 \pm 20 \ddagger$ & $18 \pm 18 \S$ \\
1 hour after meal & $76 \pm 18 \ddagger$ & $39 \pm 23 \S$ \\
2 hours after meal & $79 \pm 17 \ddagger$ & $49 \pm 25 \S$ \\
PFC (mm) & & \\
Pre-meal & $65 \pm 18$ & $63 \pm 20$ \\
Post-meal & $64 \pm 18 \ddagger$ & $25 \pm 19 \S$ \\
1 hour after meal & $76 \pm 18 \ddagger$ & $45 \pm 20 \S$ \\
2 hours after meal & $79 \pm 13 \ddagger$ & $54 \pm 23$
\end{tabular}

*BO $=$ breakfast omission; $\mathrm{BC}=$ breakfast consumption.

$\dagger$ Values are presented in mean $\pm S D, n=16$.

tSignificantly different from BC.

$\S S$ ignificantly different compared with a pre-meal. 


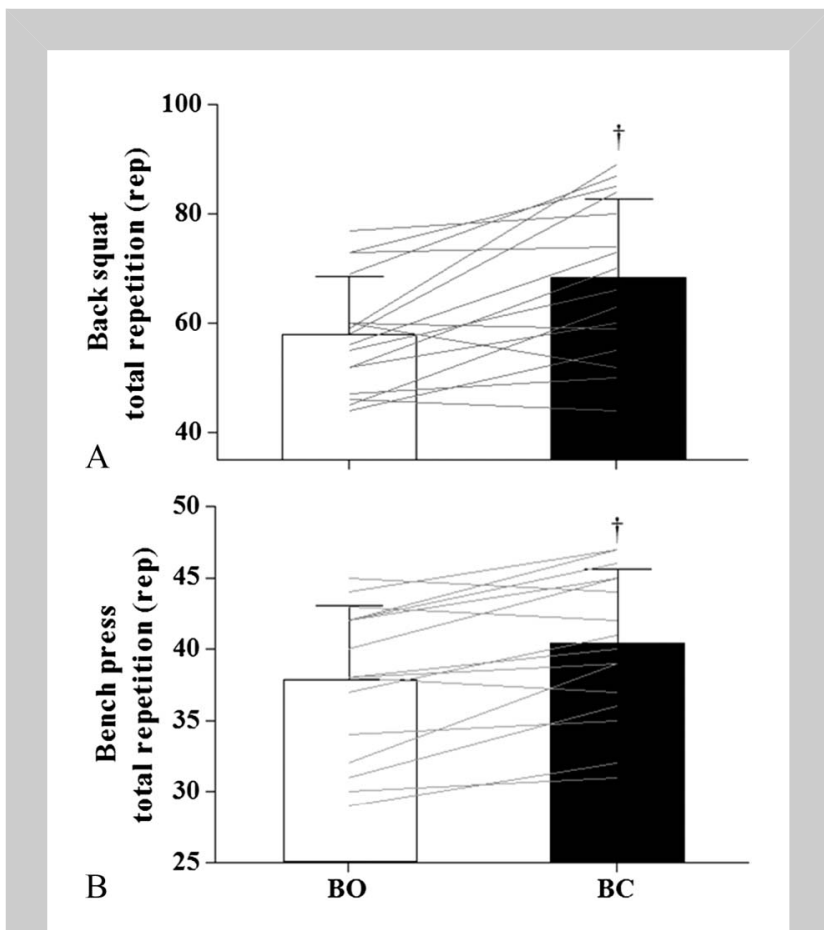

Figure 1. Total repetitions over the 4 sets for back squat $(A)$ and bench press $(B)$. Bars are mean values, vertical error bars represent $S D$, and lines represent individual subject data, $n=16$. †Significantly different from BC $(p<0.05)$. BO = breakfast omission; BC = breakfast consumption.

Subjects rated the breakfast consumed during $\mathrm{BC}$ as more pleasant (BO: $7 \pm 13 \mathrm{~mm}$; BC: $63 \pm 21 \mathrm{~mm}$; $\mathrm{ES}=2.00$; $p<$ $0.001)$ and more filling (BO: $42 \pm 21 \mathrm{~mm}$; BC: $77 \pm 14 \mathrm{~mm}$; $\mathrm{ES}=1.25 ; p=0.001)$ than the water consumed in $\mathrm{BO}$.

\section{Strength Performance}

Total repetitions completed for back squat were $15 \%$ lower during $\mathrm{BO}$, equating to a reduction of 10 repetitions over the 4 sets $(95 \% \mathrm{CI}-5$ to -15 repetitions; BO: $58 \pm 11$ repetitions; $\mathrm{BC}: 68 \pm 14$ repetitions; $\mathrm{ES}=0.98 ; p<0.001$; Figure 1A). There were trial $\left(\eta_{\mathrm{p}}^{2}=0.504 ; p<0.001\right)$, time $\left(\eta_{\mathrm{p}}^{2}=0.827 ; p<0.001\right)$, and interaction $\left(\eta_{\mathrm{p}}^{2}=0.217 ; p=\right.$ $0.027)$ effects for back squat performance. Repetitions in sets $1(\mathrm{ES}=1.15 ; p=0.001)$ and $2(\mathrm{ES}=0.90 ; p=0.008)$ were lower during $\mathrm{BO}$ compared with $\mathrm{BC}$, whereas repetitions in sets $3(\mathrm{ES}=0.16 ; p=0.54)$ and $4(\mathrm{ES}=0.40 ; p=0.13)$ were not different between trials (Figure $2 \mathrm{~A}$ ).

Total repetitions completed for bench press were $6 \%$ lower during $\mathrm{BO}$, equating to a reduction of 3 repetitions over the 4 sets $(95 \% \mathrm{CI}-1$ to -4 repetitions; BO: $38 \pm 5$ repetitions; BC: $40 \pm 5$ repetitions; ES: 1.06; $p<0.001$; Figure $1 \mathrm{~B})$. For bench press performance, there were trial $\left(\eta_{\mathrm{p}}^{2}=0.544 ; p=0.001\right)$ and time $\left(\eta_{\mathrm{p}}^{2}=0.892 ; p<0.001\right)$ effects but no interaction effect $\left(\eta_{\mathrm{p}}^{2}=0.078 ; p=0.297\right)$. Similar to back squat, repetitions in sets $1(\mathrm{ES}=0.88 ; p=$ $0.009)$ and $2(\mathrm{ES}=0.70 ; p=0.038)$ were lower during $\mathrm{BO}$

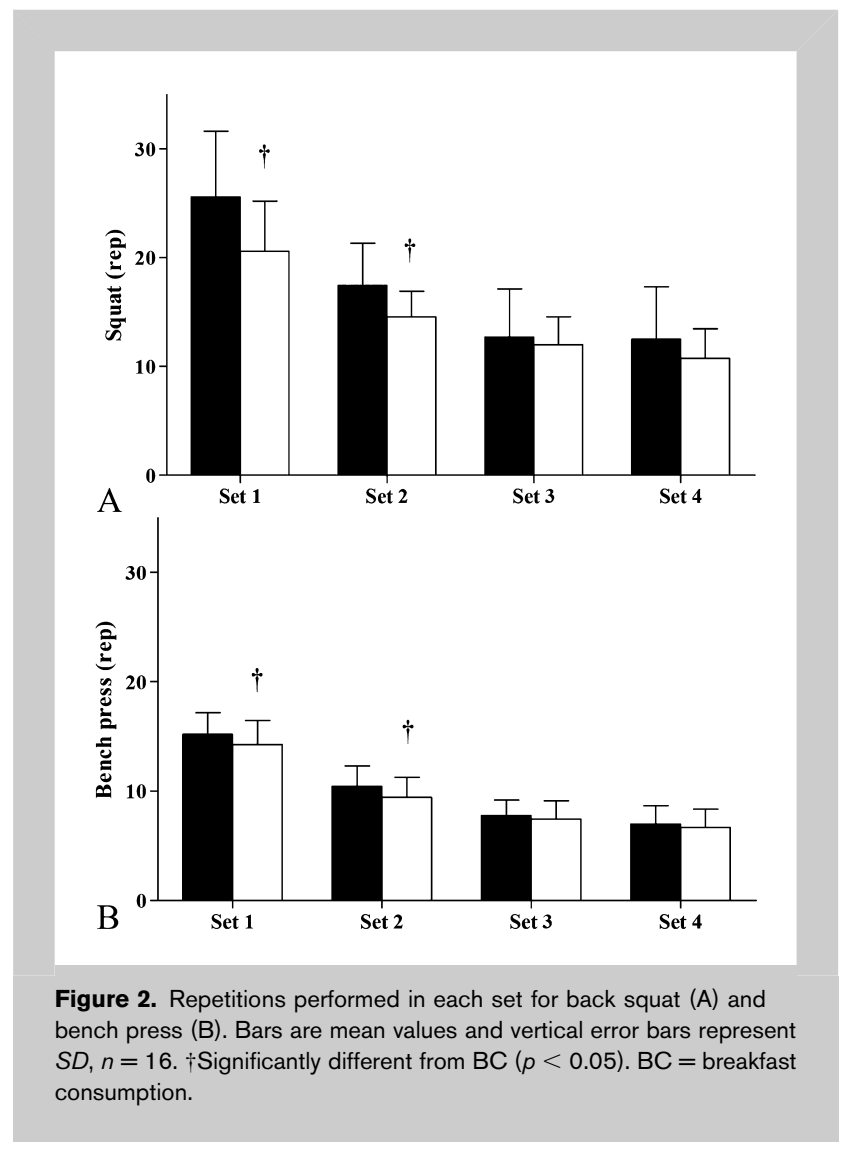

compared with $\mathrm{BC}$, whereas repetitions in sets $3(\mathrm{ES}=0.30$; $p=0.24)$ and $4(\mathrm{ES}=0.18 ; p=0.46)$ were not different between trials (Figure 2B).

\section{Subjective Appetite Sensations}

There were trial $\left(\eta_{\mathrm{p}}^{2}<0.881 ; p<0.001\right)$, time $\left(\eta_{\mathrm{p}}^{2}<0.687\right.$; $p$ $<0.001)$, and interaction $\left(\eta_{\mathrm{p}}^{2}<0.614 ; p<0.001\right)$ effects for all subjective appetite sensations (Table 2). At all post-meal time points, fullness was greater, whereas hunger, DTE, and PFC were all lower after BC compared with BO $(p<0.001)$. Compared with pre-meal values, the breakfast during $\mathrm{BC}$ decreased hunger, DTE, and PFC, and increased fullness immediately and 1 hour after a meal $(p \leq 0.001)$, with the effects for fullness and DTE persisting to 2 hours after a meal $(p \leq 0.043)$. By contrast, compared with pre-meal, the water-only breakfast in BO increased fullness immediately after a meal $(p=0.009)$ and increased hunger and DTE 2 hours after a meal $(p \leq 0.002)$.

\section{Discussion}

This study is the first to report the effect of an ecologically valid high-carbohydrate pre-exercise breakfast on subsequent resistance exercise performance $\sim 2$ hours later. The main findings of this study were that subjects completed $\sim 15 \%$ less repetitions of back squat and $\sim 6 \%$ less repetitions of bench press when they omitted breakfast compared with

4 Journal of Strength and Conditioning Research 
when they consumed the high-carbohydrate breakfast (providing $1.5 \mathrm{~g}$ of carbohydrate $/ \mathrm{kg}$ body mass). This suggests that a high-carbohydrate meal might be a beneficial preexercise strategy to augment an increased training volume at a morning resistance exercise session, at least in habitual breakfast consumers.

Although there is a wealth of literature suggesting that pre-exercise carbohydrate/energy intake enhances prolonged endurance performance, little is known about how resistance-type exercise performance is influenced by preexercise nutrition. Thus, this study provides novel data suggesting that resistance exercise performance can be enhanced by the consumption of a high-carbohydrate preexercise meal. This finding contrasts the results of the only other study to investigate the isolated effects of pre-exercise carbohydrate/energy intake on resistance exercise performance (8). These disparate results might be explained by the nature of the resistance exercise performed, where different mechanisms are likely to limit performance. Performance in the study by Fairchild et al. (8) was measured through 3 maximal efforts on an isokinetic dynamometer, with this performance unlikely to be dependent on muscle glycogen availability. By contrast, the higher volume nature of the exercise used in this study, which represents a more ecologically valid resistance training scenario, is more likely to rely on glycogen as a fuel source (22), potentially explaining the observed results.

Indeed, some evidence supports the notion that preresistance exercise carbohydrate intake/glycogen stores might be an important determinant of performance. Leveritt and Abernethy (23) reported that a muscle glycogendepleting regimen (cycling exercise followed by 2 days of low-carbohydrate diet) produced a $\sim 20 \%$ reduction in the number of repetitions completed in 3 sets of back squat to fatigue. Interestingly, Leveritt and Abernethy (23) also measured performance in 5 sets of 5 repetitions of isokinetic dynamometry, observing no difference between conditions. This suggests, as mentioned above, that pre-exercise glycogen stores might play a more important role where the volume of work performed is higher. Similarly, Haff et al. (16) and Oliver et al. (28) reported that performance during resistance exercise a few hours after a bout of glycogen-depleting exercise was enhanced when carbohydrate was provided between the 2 exercise bouts. Although muscle glycogen was not measured in these studies, the ingestion of carbohydrate in 1 trial, but not the other, would be expected to alter glycogen resynthesis and glycogen content at the start of the second bout of exercise. Mitchell et al. (25) manipulated muscle glycogen before a bout of resistance exercise with the subjects performing cycling exercise, followed by 48 hours of either high- or low-carbohydrate intake. In contrast to previous studies $(15,28)$, performance in 5 sets to fatigue $(\sim 10-15$ repetitions) of squat, leg press, and leg extension was not affected by this pre-exercise regimen. Although an explanation for the disparity in findings between studies re- mains elusive, the lack of a direct measure of muscle glycogen means there is uncertainty about whether muscle (or indeed liver) glycogen stores were different between conditions. On balance, it would seem that starting exercise with high glycogen stores or after a high-carbohydrate intake is the most prudent approach to maximize resistance exercise performance, with the findings of the current study perhaps providing further evidence of this.

Muscle glycogen seems to be an important fuel source during resistance-type exercise (21), with studies reporting muscle glycogen depletion by up to $40 \%$ after a single bout of resistance exercise $(12,13,30,32)$. For example, Robergs et al. (30) reported that 6 sets of 6 repetitions of leg extension at $70 \% 1 \mathrm{RM}$ caused a $38 \%$ reduction in the glycogen content of the vastus lateralis. It is well documented that although type II muscle fibers have greater glycogen stores compared with type I fibers (11), the rate of glycogenolysis during highintensity exercise is also greater $(11,18,34)$. Resistance exercise augments a similar response, with selectively greater muscle glycogen use in type II vs. type I muscle fibers $(19,30)$. If, as suggested by Lambert and Flynn (21), the depletion of muscle glycogen is a contributing factor to the generation of fatigue during resistance exercise, then increasing pre-exercise glycogen stores might have the potential to enhance performance.

Consumption of a high-carbohydrate meal after an overnight fast has been shown to produce a small, but potentially meaningful, increase in the skeletal muscle glycogen content (3), which may account for the enhanced performance in the $\mathrm{BC}$ trial. Indeed, the fact that carbohydrate intake after an overnight fast only induces a small increase in muscle glycogen might go some way to explain the pattern of results in this study. For both back squat and bench press, repetitions completed were greater during $\mathrm{BC}$ when compared with $\mathrm{BO}$ in the first 2 sets alone. It is possible that the extra repetitions performed in sets 1 and 2 used any additional glycogen present at the start of the exercise in the $\mathrm{BC}$ trial, meaning that by the time subjects performed sets 3 and 4 , the muscle glycogen content was similar between trials. In addition to muscle glycogen, the consumption of breakfast in BC would have substantially increased liver glycogen when compared with BO $(16,27)$. Although liver glycogen seems to be an important energy store used during endurance exercise (10), its relevance to resistance-type exercise performance is unknown. However, in the absence of an exogenous carbohydrate source, liver glycogenolysis helps to maintain blood glucose levels. Provision of exogenous carbohydrate during resistance exercise has been shown to attenuate muscle glycogen use (12) and increase performance $(14,22)$. Therefore, it is possible that starting exercise with greater liver glycogen stores might mean a greater supply of glucose to the working muscle from blood glucose, reducing the reliance on muscle glycogen and enhancing performance capabilities. 
Although the blood glucose level was not measured in this study, the breakfast used in the BC trial was very similar to those used in previous published studies $(4,5)$. These previous studies report acute increases in plasma glucose and insulin concentrations, giving us confidence that the same effects were apparent in this study. Although the provision of carbohydrate in the breakfast and the subsequent effects on glycogen stores is the most likely cause of the enhanced performance, other non-nutrient-specific mechanisms might also be responsible for the observed effects. The breakfast in the $\mathrm{BC}$ trial suppressed appetite after consumption, with hunger, DTE, and PFC all lower, as well as fullness higher in $\mathrm{BC}$ compared with $\mathrm{BO}$ at all time points after eating. Although speculative, it is possible that the increase in appetite in the BO trial induced some level of discomfort/distraction that might have reduced exercise performance. Future experiments should attempt to separate the performance effects of nutrient provision from those of the appetite suppression induced by eating.

Although this study demonstrates a pre-exercise meal (i.e., breakfast) enhances resistance exercise performance, the results are not without limitation. Although the purpose of this study was to examine the impact of an ecologically valid breakfast on resistance exercise performance, this meant, by design, that while the study included a control trial (i.e., the BO trial), the results observed could be caused by the subject's knowledge of whether they consumed breakfast or not. It is possible that the breakfast in the $\mathrm{BC}$ trial might have acted as a placebo to enhance performance. Conversely, given that subjects were habitual breakfast consumers, the BO trial likely represented a deviation from their normal practices, which might have limited performance. Therefore, the decreased performance could also have been due to psychological factors associated with subjects knowing they were consuming/omitting a pre-exercise meal/ breakfast. In line with this, Mears et al. (24) recently reported that short-duration cycling time-trial performance $(\sim 20$ minutes) was similarly enhanced by placebo (a very low energy thickened breakfast drink) and highcarbohydrate (the same breakfast drink plus $2 \mathrm{~g}$ of carbohydrate/ $\mathrm{kg}$ body mass) breakfasts compared with a water-only breakfast. This suggests that in some situations, breakfast/a pre-exercise meal might act as a placebo that can enhance performance, which is something future studies should investigate with resistance exercise performance.

\section{Practical Applications}

The results of this study demonstrate that, for athletes, consumption of a pre-exercise breakfast meal (1.5 g of carbohydrate $/ \mathrm{kg}$ body mass and $\sim 20 \%$ from total daily energy requirements) might enhance resistance exercise performance, at least among habitual breakfast consumers. This might have implications for the volume of exercise completed and possibly the subsequent adaptive response to exercise training. Therefore, these results suggest that, at least for exercise sessions where subjects exercise to failure, it might be advisable to recommend the consumption of a high-carbohydrate meal before exercise, particularly if the training session is undertaken in the morning. However, given the paucity of data in this area, future studies should explore optimal amounts and timings of pre-exercise meals to maximize resistance-type exercise performance.

\section{ACKNowledgments}

The authors thank all subjects who gave their time and commitment to complete this study. This study was funded by the Research Management and Monitoring Research FundPG170-2014B and the University of Malaya Research GrantUM.0000215/HRU.OP/RU027-2015. L.J. James is part of the National Institute for Health Research (NIHR) Leicester Biomedical Research Center, which is a partnership between University Hospitals of Leicester NHS Trust, Loughborough University, and the University of Leicester. This report is an independent research by the National Institute for Health Research. The views expressed in this publication are those of the authors and not necessarily those of the NHS, the National Institute for Health Research, or the Department of Health. This study does not constitute endorsement of the product by the authors or the National Strength and Conditioning Association.

\section{REFERENCES}

1. Bawden, SJ, Stephenson, MC, Ciampi, E, Hunter, K, Marciani, L, Spiller, RC, et al. A low calorie morning meal prevents the decline of hepatic glycogen stores: A pilot in vivo ${ }^{13} \mathrm{C}$ magnetic resonance study. Food Funct 5: 2237-2242, 2014.

2. Burke, LM, Hawley, JA, Wong, SH, and Jeukendrup, AE. Carbohydrates for training and competition. J Sports Sci29(Suppl 1): S17-S27, 2011.

3. Chryssanthopoulos, C, Williams, C, Nowitz, A, and Bogdanis, G. Skeletal muscle glycogen concentration and metabolic responses following a high glycaemic carbohydrate breakfast. J Sports Sci 22: 1065-1071, 2004.

4. Clayton, DJ, Burrell, K, Mynott, G, Creese, M, Skidmore, N, Stensel, DJ, et al. Effect of 24-h severe energy restriction on appetite regulation and ad libitum energy intake in lean men and women. $\mathrm{Am}$ $J$ Clin Nutr 104: 1545-1553, 2016.

5. Clayton, DJ, Creese, M, Skidmore, N, Stensel, DJ, and James, LJ. No Effect of $24 \mathrm{~h}$ severe energy restriction on appetite regulation and ad-libitum energy intake in overweight and obese males. Int $J$ Obes (Lond) 40: 1662-1670, 2016.

6. Clayton, DJ and James, LJ. The effect of breakfast on appetite regulation, energy balance and exercise performance. Proc Nutr Soc 75: 319-327, 2016

7. Coyle, EF, Coggan, AR, Hemmert, MK, Lowe, RC, and Walters, TJ. Substrate usage during prolonged exercise following a preexercise meal. J Appl Physiol 59: 429-433, 1985.

8. Fairchild, TJ, Dillon, P, Curtis, C, and Dempsey, AR. Glucose ingestion does not improve maximal isokinetic force. $J$ Strength Cond Res 30: 194-199, 2016.

9. Flint, A, Raben, A, Blundell, JE, and Astrup, A. Reproducibility, power and validity of visual analogue scales in assessment of appetite sensations in single test meal studies. Int J Obes Relat Metab Disord 24: 38-48, 2000.

10. Gonzalez, JT, Fuchs, CJ, Betts, JA, and van Loon, LJC. Liver glycogen metabolism during and after prolonged endurance-type exercise. Am J Physiol Endocrinol Metab 311: E543-E553, 2016. 
11. Greenhaff, PL, Nevill, ME, Soderlund, K, Bodin, K, Boobis, LH, Williams, C, et al. The metabolic responses of human type I and II muscle fibres during maximal treadmill sprinting. J Physiol (Lond) 478(Pt 1): 149-155, 1994.

12. Haff, GG, Koch, AJ, Potteiger, JA, Kuphal, KE, Magee, LM, Green, $\mathrm{SB}$, et al. Carbohydrate supplementation attenuates muscle glycogen loss during acute bouts of resistance exercise. Int J Sport Nutr Exerc Metab 10: 326-339, 2000.

13. Haff, GG, Lehmkuhl, MJ, McCoy, LB, and Stone, MH. Carbohydrate supplementation and resistance training. $J$ Strength Cond Res 17: 187-196, 2003.

14. Haff, GG, Schroeder, CA, Koch, AJ, Kuphal, KE, Comeau, MJ, and Potteiger, JA. The effects of supplemental carbohydrate ingestion on intermittent isokinetic leg exercise. J Sports Med Phys Fitness 41: 216-222, 2001.

15. Haff, GG, Stone, MH, Warren, BJ, Keith, R, Johnson, RL, Nieman, DC, et al. The effect of carbohydrate supplementation on multiple sessions and bouts of resistance exercise. J Strength Cond Res 13: 111, 1999.

16. Hawley, JA and Burke, LM. Effect of meal frequency and timing on physical performance. Br J Nutr 77(Suppl 1): S91-S103, 1997.

17. Jacobs, I, Kaiser, P, and Tesch, P. Muscle strength and fatigue after selective glycogen depletion in human skeletal muscle fibers. Eur $J$ Appl Physiol Occup Physiol 46: 47-53, 1981.

18. Judelson, DA, Maresh, CM, Yamamoto, LM, Farrell, MJ, Armstrong, LE, Kraemer, WJ, et al. Effect of hydration state on resistance exercise-induced endocrine markers of anabolism, catabolism, and metabolism. J Appl Physiol 105: 816-824, 2008.

19. Koopman, R, Manders, RJF, Jonkers, RAM, Hul, GBJ, Kuipers, H, and van Loon, LJC. Intramyocellular lipid and glycogen content are reduced following resistance exercise in untrained healthy males. Eur J Appl Physiol 96: 525-534, 2006.

20. Kulik, JR, Touchberry, CD, Kawamori, N, Blumert, PA, Crum, AJ, and Haff, GG. Supplemental carbohydrate ingestion does not improve performance of high-intensity resistance exercise. $J$ Strength Cond Res 22: 1101-1107, 2008.

21. Lambert, $\mathrm{CP}$ and Flynn, MG. Fatigue during high-intensity intermittent exercise: Application to bodybuilding. Sports Med 32: 511-522, 2002.

22. Lambert, CP, Flynn, MG, Boone, JB, Michaud, TJ, and RodriguezZayas, J. Effects of carbohydrate feeding on multiple-bout resistance exercise. J Strength Cond Res 5: 192-197, 1991.

23. Leveritt, $\mathrm{M}$ and Abernethy, PJ. Effects of carbohydrate restriction on strength performance. J Strength Cond Res 13: 52-57, 1999.
24. Mears, SA, Dickinson, K, Bergin-Taylor, K, Dee, R, Kay, J, and James, LJ. Perception of breakfast ingestion enhances high intensity cycling performance. Int J Sports Physiol Perform 13 504-509, 2017.

25. Mitchell, JB, DiLauro, PC, Pizza, FX, and Cavender, DL. The effect of preexercise carbohydrate status on resistance exercise performance. Int J Sport Nutr 7: 185-196, 1997.

26. Neufer, PD, Costill, DL, Flynn, MG, Kirwan, JP, Mitchell, JB, and Houmard, J. Improvements in exercise performance: Effects of carbohydrate feedings and diet. J Appl Physiol 62: 983-988, 1987.

27. Nilsson, LH and Hultman, E. Liver glycogen in man-the effect of total starvation or a carbohydrate-poor diet followed by carbohydrate refeeding. Scand J Clin Lab Invest 32: 325-330, 1973.

28. Oliver, JM, Almada, AL, Van Eck, LE, Shah, M, Mitchell, JB, Jones, MT, et al. Ingestion of high molecular weight carbohydrate enhances subsequent repeated maximal power: A randomized controlled trial. PLoS One 11: e0163009, 2016.

29. Ormsbee, MJ, Bach, CW, and Baur, DA. Pre-exercise nutrition: The role of macronutrients, modified starches and supplements on metabolism and endurance performance. Nutrients 6: 1782-1808, 2014.

30. Robergs, RA, Pearson, DR, Costill, DL, Fink, WJ, Pascoe, DD, Benedict, MA, et al. Muscle glycogenolysis during differing intensities of weight-resistance exercise. J Appl Physiol 70: 17001706, 1991.

31. Slater, G and Phillips, SM. Nutrition guidelines for strength sports: Sprinting, weightlifting, throwing events, and bodybuilding. J Sports Sci 29(Suppl 1): S67-S77, 2011.

32. Tesch, PA, Ploutz-Snyder, LL, Yström, L, Castro, MJ, and Dudley, GA. Skeletal muscle glycogen loss evoked by resistance exercise. J Strength Cond Res 12: 67-73, 1998.

33. Veasey, RC, Haskell-Ramsay, CF, Kennedy, DO, Tiplady, B, and Stevenson, EJ. The effect of breakfast prior to morning exercise on cognitive performance, mood and appetite later in the day in habitually active women. Nutrients 7: 5712-5732, 2015.

34. Vøllestad, NK, Tabata, I, and Medbø, JI. Glycogen breakdown in different human muscle fibre types during exhaustive exercise of short duration. Acta Physiol Scand 144: 135-141, 1992.

35. Zullig, K, Ubbes, VA, Pyle, J, and Valois, RF. Self-reported weight perceptions, dieting behavior, and breakfast eating among high school adolescents. $J$ Sch Health 76: 87-92, 2006. 